\title{
New Interpolator and Data Detector for Full Digital BPSK Demodulator
}

\author{
Caio Gomes de Figueredo \\ Teleinfomatic Engineering \\ Department (DETI) \\ Federal University of Ceara (UFC) \\ Email:caiofigueredo@gmail.com
}

\author{
Antonio Macilio Pereira de Lucena \\ National Institute of Space \\ Research (INPE) and Fortaleza \\ University (UNIFOR) \\ Email: macilio@roen.inpe.br
}

\author{
J. C. M. Mota \\ Signal and Information \\ Processing Group (GPSI) \\ Federal University of Ceara (UFC) \\ Email:mota@gtel.ufc.br
}

\begin{abstract}
This work presents a new structure for an alldigital BPSK demodulator developed for space communications that performs simultaneously the sampling and down conversion from intermediate frequency signal to the baseband signal. A new interpolator is proposed in order to simplify the demodulator implementation. This interpolator correlates the samples of the output signal in such way that it was necessary to design a new data detector appropriate to process the samples corrupted by gaussian and colored noise. The effects of the new interpolation at the noise are analyzed as well as the way it affects the whole demodulator performance. Simulations were performed and the results are presented to confirm theoretical analysis.
\end{abstract}

\section{INTRODUCTION}

Satellite telecommand links demand for higher rate capabilities had increased over the past years, reaching transmission rates up to $2048 \mathrm{Mb} / \mathrm{s}$. For such high rates transmission systems, the Consultative Comitte for Space Data Systems (CCSDS) recommends the utilization of a BPSK modulation combined with a rectangular pulse shaping [1]. Despite its large bandwidth, making its use discarded for most applications, this kind of pulse shaping is very often employed in spatial telecommand modems due to its simplicity, a mandatory requisite for onboard systems.

Regarding these recommendations, this work presents a new interpolator and data detector applied to an all-digital BPSK demodulator for a satellite telecommand application. Bandpass sampling technique [2, 3], that simultaneously down converts the signal from bandpass to baseband frequency, was employed. This approach reduces the complexity of the system because it does not use analogic frequency convertors, which are often subject to imperfections like misadjustments and nonlinearities, preserving the system performance as good as the analogic equivalent. A sub-Nyquist sampling rate [2] is used at this sampling process, which means that the received signal is sampled in a rate lower than the Nyquist rate, with respect to the carrier frequency. This technique allows a reduction in the volume of data to be processed, another important requisite for an onboard system.

One problem that emerges from this sampling process is the lost of samples due to the interleaving of in-phase and quadrature channel samples, an issue solved using digital interpolation. The utilization of a rectangular pulse shape makes this interpolation task much simpler than usual, avoiding the need of more sophisticated filtering operations. The main contributions of this paper are: the proposal and mathematical analysis of a new interpolator, the design of the maximum likelihood (ML) symbol detector for the interpolated signal and the analytical determination of the system error rate. Computational simulation results are also presented in order to confirm some analytical outcomes.

This paper is organized as follows: in section II, the general model of the demodulator is presented and the main signals involved are briefly described. In section III, the down convertion process to baseband frequency is investigated and then, in section IV, the interpolation technique is presented, starting by the description of the general structure of the interpolator, followed by an investigation on how this interpolation affects the noise. The project of the optimum detector is presented in the Section V. The bit error rate of the system is evaluated in the Section VI. In the Section VII, simulation results are shown and finally, in the Section VIII, we have the conclusion.

\section{Demodulator Model}

Figure 1 shows the demodulator simplified block diagram.

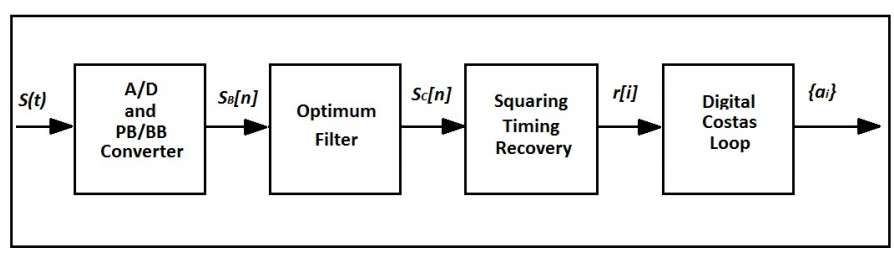

Fig. 1. Demodulator Model.

Before the signal entering the demodulator, it passes through an IF filter centered at $f_{F I}=70 \mathrm{MHz}$, having a bandwidth equal to $f_{s} / 2$, where $f_{s}$ is the sampling rate. This filter is modeled as a rectangular window, in the frequency domain, and its bandwidth is large enough to avoid distortions at the received signal.

As it is shown in Figure 1, the signal $s(t)$ is discretized and converted to baseband by the $\mathrm{A} / \mathrm{D}$ and $\mathrm{PB} / \mathrm{BB}$ converter block. The resulting baseband signal $s_{B}[n]$ is processed by the optimum filter that implements the required metric, from the samples of the symbol, for the optimum detection by the ML criterion. The squaring recovery block [4] corrects any time error by interpolating the $s_{C}[n]$ signal in order to choose the best sample. The last block implements a digital Costas loop 
[5] that estimates and corrects the phase error and performs the bit decision.

The Figure 2 details the functionalities of the A/D and $\mathrm{PB} / \mathrm{BB}$ converter block.

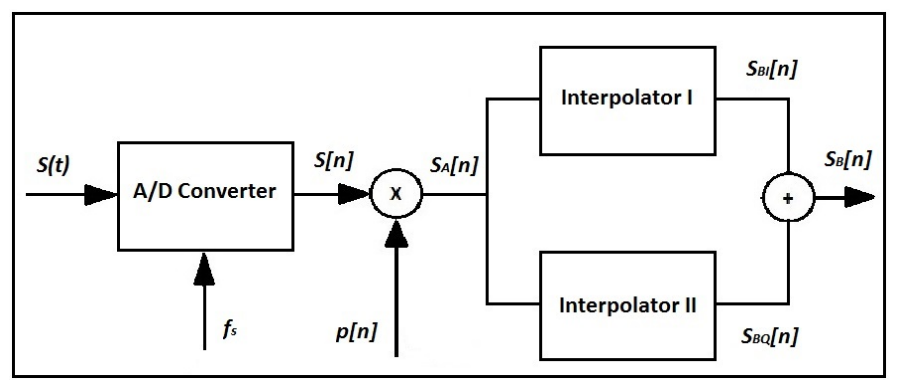

Fig. 2. Converter Model.

The signal $s(t)$, at the input of the A/D converter, can be expressed by

$$
s(t)=A(t) \cos \left[2 \pi\left(f_{F I}+f_{d}\right) t+\varphi\right]+w(t),
$$

where the baseband signal $A(t)$ is modeled as

$$
A(t)=\sum_{i} a_{i} g(t-i T-\tau),
$$

$a_{i}$ are the BPSK transmitted symbols, $T$ is the symbol period, $g(t)$ is the baseband pulse defined as a rectangular window having a duration of $T$ seconds and amplitude $\sqrt{\frac{T_{s}}{T}}$, where $T_{s}=\frac{1}{f_{s}}$. The frequency offset, the phase error offset and the time delay are $f_{d}, \varphi$ and $\tau$, respectively. The narrow bandwidth gaussian noise signal $w(t)$ has the following power spectral density:

$$
S_{w}(f)=\left\{\begin{array}{rll}
N_{o} / 2, & \text { if } & \left|f-f_{F I}\right| \leq f_{s} / 4 \\
0, & \text { if } & \left|f-f_{F I}\right|>f_{s} / 4
\end{array}\right.
$$

As it is shown in Fig. 2, $s(t)$ is sampled at the A/D converter, resulting in the signal $s[n]$, and then multiplied by the sequence $p[n]$, generating $s_{A}[n]$ which, as we will see later, consists of interleaved samples of the real and imaginary parts of the baseband signal plus noise. Next, interpolation is used to retrieve that lost samples of the real and imaginary components of the baseband equivalent signal, denoted by $s_{B I}[n]$ and $s_{B Q}[n]$, respectively.

As synchronization issues are not the main subject of the paper, for most part of mathematical analysis that will be done and for the results, it will be considered perfect synchronization. Although we made this assumption, it is important to remark that the demodulator also shows a good performance in the presence of synchronization errors, exhibiting just a little degradation. In the next sections, we proceed with the mathematical analysis, detailing all the processing in each block at the diagram of the Fig. 2.

\section{Determination OF THE BASEbAND SignAL}

To avoid aliasing at the sampling process, we should compute the value of the frequency $f_{s}$ using the following equation [6], where the highest frequency of the positive spectrum of $s(t)$ is $f_{u}$ and the lowest is $f_{l}$ :

$$
\frac{2 f_{u}}{q} \leq f_{s} \leq \frac{2 f_{l}}{q-1}
$$

and $q$ is an integer number satisfying the condition:

$$
1 \leq q \leq \operatorname{int}\left(\frac{f_{u}}{f_{u}-f_{l}}\right)
$$

$\operatorname{int}(x)$ means the integer part of $x$. The discrete signal $s[n]$ is expressed by:

$$
s[n]=\Re\left\{A[n] e^{j\left[2 \pi\left(\frac{f_{F I}+f_{d}}{f_{s}}\right) n+\varphi\right]}\right\}+w[n]
$$

where $A[n]$ and $w[n]$ are the discrete versions of the signals $A(t)$ and $w(t)$ and $\Re\{x\}$ represents the real part of $x$. If we use $f_{s}=4 f_{F I} /(2 K+1), K \in \mathbb{N}$, a special case of Equation (4), in (6) and remembering that $\omega_{d}=2 \pi f_{d} T_{s}, s[n]$ becomes:

$$
s[n]=\Re\left\{A[n] e^{j\left(\omega_{d} n+\varphi\right)} e^{j\left(K \pi+\frac{\pi}{2}\right) n}\right\}+w[n]
$$

At this particular case, we used $K=3$ and, as a consequence, $f_{s}=40 M H z$ and $w[n]$ is white. Substituting the values at the above equation:

$$
s[n]=\Re\left\{A[n] e^{j\left(\omega_{d} n+\varphi\right)} e^{j\left(\frac{7 \pi}{2}\right) n}\right\}+w[n]
$$

As it is ilustrated in Figure 2, the signal down conversion to baseband frequency is realized by multiplying it by $p[n]=$ $\cos (7 \pi n / 2)-\sin (7 \pi n / 2)$. Hence, the baseband signal is given by:

$$
\begin{aligned}
& s_{A}[n]=A[n] \cos \left[\left(\frac{7 \pi}{2}+\omega_{d}\right) n+\varphi\right] \cos \left(\frac{7 \pi n}{2}\right) \\
& -A[n] \cos \left[\left(\frac{7 \pi}{2}+\omega_{d}\right) n+\varphi\right] \sin \left(\frac{7 \pi n}{2}\right)+z[n]
\end{aligned}
$$

where $z[n]$ is a random process generated by the multiplication of $w[n]$ by $p[n]$. Using trigonometrical identities to develop (9), $s_{A}[n]$ becomes:

$$
\begin{gathered}
s_{A}[n]=\frac{A[n]}{2}\left\{\cos \left(\omega_{d} n+\varphi\right)\left((-1)^{n}+1\right)\right. \\
\left.-\sin \left(\omega_{d} n+\varphi\right)\left((-1)^{n}-1\right)\right\}+z[n]
\end{gathered}
$$

Simplifying a little more, it can be shown that $s_{A}[n]$ is expressed as follows:

$$
s_{A}[n]= \begin{cases}A[n] \cos \left(\omega_{d} n+\varphi\right)+z[n], & \text { if } n \text { even, } \\ A[n] \sin \left(\omega_{d} n+\varphi\right)+z[n], & \text { if } n \text { odd }\end{cases}
$$

From this last equation, it is clear the interleaving of samples that was described earlier. Every even sample of $s_{A}[n]$ consists of the baseband signal $A[n]$ multiplied by the cosine whose argument depends on the frequency and phase error offsets. For every odd sample, it is exactly the same signal multiplied by a sine.

Next, the signal $s_{A}[n]$ passes through two interpolators, each one retrieving those lost samples of in-phase and quadrature components, to generate the signals $s_{B I}[n]$ and $s_{B Q}[n]$. It is important to remark that both branches use the same interpolation technique, differing only on which sample, odd or even, to retrieve. Based on that, it is sufficient to discuss the interpolation for just one branch of the demodulator, ignoring 
the other one. At this paper, once we are dealing with a BPSK modulation scheme, is analyzed the in-phase branch.

As discussed earlier, synchronization issues are out of the scope of this paper, since it does not affect the interpolation operation. Hence, making $\omega_{d}=\varphi=0$, Equation (11) can be simplified to:

$$
s_{A}[n]=\left\{\begin{aligned}
A[n]+z[n], & \text { if } n \text { even, } \\
z[n], & \text { if } n \text { odd. }
\end{aligned}\right.
$$

Now, our immediate problem is how to recover the odd sample of the signal $A[n]$. There are several possibilities [3], including linear and non-linear interpolation. In the next section, we present the proposed interpolation technique and analyze the statistical properties of the output signal $s_{B I}[n]$.

\section{INTERPOLATION}

\section{A. Proposed Technique}

Considering the baseband pulse is rectangular, the samples of the signal $A[n]$ are, for each symbol, constants and equal to $\sqrt{\frac{T_{s}}{T}}$ or $-\sqrt{\frac{T_{s}}{T}}$, depending on the trasmitted information. Therefore, an obvious interpolation technique should be to make the lost odd sample equal to the previous even sample. Such operation is described by the equation:

$$
s_{B I}[n]=\left\{\begin{aligned}
A[n]+z[n], & \text { if } n \text { even, } \\
A[n-1]+z[n-1], & \text { if } n \text { odd. }
\end{aligned}\right.
$$

For a given symbol, the signal $s_{B I}[n]$ may be expressed in a more simplified way:

$$
s_{B I}[n]=\hat{A}[n]+v[n],
$$

where $\hat{A}[n]$ is an estimate of the baseband transmitted signal $A[n]$ at the interpolator output, and it is given by the expression:

$$
\hat{A}[n]=\left\{\begin{array}{rc}
A[n], & \text { if } n \text { even, } \\
A[n-1], & \text { if } n \text { odd. }
\end{array}\right.
$$

and $v[n]$ is the colored noise, represented by:

$$
v[n]=\left\{\begin{array}{rc}
z[n], & \text { if } n \text { even, } \\
z[n-1], & \text { if } n \text { odd. }
\end{array}\right.
$$

The statistical characterization of the noise $v[n]$ is made in the next subsection.

\section{B. Interpolated Noise}

Considering the power spectrum density of $w(t)$, at the IF filter output, given by Equation (3), the random process $w[n]$ is gaussian, white and its autocorrelation function is expressed by [3]:

$$
R_{w}[m]=\frac{N_{o}}{2 T_{s}} \delta[m]
$$

where $\delta[$.$] is the unit impulse function. After the multiplication$ of the received signal by $p[n]$, the noise factor $z[n]=w[n] p[n]$ is also gaussian and white, and its autocorrelation function is identical to $w[n]$. The interpolator output, taking in account only the noise term, is modeled as:

$$
v[n]=\left\{\begin{aligned}
z[n], & \text { if } n \text { even, } \\
z[n-1], & \text { if } n \text { odd. }
\end{aligned}\right.
$$

which can be rewritten in a single expression:

$$
v[n]=\frac{z[n]}{2}[1+\cos (\pi n)]+\frac{z[n-1]}{2}[1-\cos (\pi n)]
$$

The autocorrelation function of $v[n]$ is:

$$
R_{v}[n, m]=E\{v[n] v[n+m]\}
$$

and $E\{x\}$ is the mathematical expectation of $x$. Developing (20) using the representation of $v[n]$ in (19), the expression for $R_{v}[n, m]$ becomes:

$$
\begin{aligned}
& R_{v}[n, m]=\frac{1}{4} E\{z[n] z[n+m](1+\cos (\pi n)[1+\cos \pi(n+m)]\} \\
& \quad+\frac{1}{4} E\{z[n] z[n+m-1](1+\cos (\pi n)[1-\cos \pi(n+m)]\} \\
& \quad+\frac{1}{4} E\{z[n-1] z[n+m](1-\cos (\pi n)[1+\cos \pi(n+m)]\} \\
& +\frac{1}{4} E\{z[n-1] z[n+m-1](1-\cos (\pi n)[1-\cos \pi(n+m)]\}
\end{aligned}
$$

From the equation above, $R_{v}[n, m]$ consists in an addition of four mathematical expectations terms, each of them can be easily simplified using trigonometrical identities. This simplified expression for the autocorrelation function of $v[n]$ is:

$$
\begin{gathered}
R_{v}[n, m]=\frac{N_{o}}{4 T_{s}} \delta[m](1+\cos \pi n)+\frac{N_{o}}{4 T_{s}} \delta[m-1](1+\cos \pi n) \\
+\frac{N_{o}}{4 T_{s}} \delta[m+1](1-\cos \pi n)+\frac{N_{o}}{4 T_{s}} \delta[m](1-\cos \pi n)
\end{gathered}
$$

Rearranging once more these terms, the final expression becomes:

$$
\begin{aligned}
R_{v}[n, m]= & \frac{N_{o}}{2 T_{s}} \delta[m]+\frac{N_{o}}{4 T_{s}} \delta[m-1](1+\cos \pi n) \\
& +\frac{N_{o}}{4 T_{s}} \delta[m+1](1-\cos \pi n)
\end{aligned}
$$

Examining this equation, it is easy to conclude that the random process $v[n]$ is cyclostationary with a discrete period of two samples. Instead of using (22), it is common practice [7] to work with the average autocorrelation function which is defined as the average of the autocorrelation function over one period. Applying this procedure, Equation (22) reduces to:

$$
\bar{R}_{v}[m]=\frac{N_{o}}{2 T_{s}} \delta[m]+\frac{N_{o}}{4 T_{s}} \delta[m-1]+\frac{N_{o}}{4 T_{s}} \delta[m+1]
$$

From the above expression, the conclusion drawn is that $v[n]$ is colored. In this case, when the noise is not white, the classical receiver structure with matched filter for implementation of ML detection is not applicable. It is necessary to design a new optimum ML detector considering the noise characteristics. 


\section{Detector Design}

The samples of $s_{B I}[n]$ conveys the infomation of which symbol was sent, hence it is essential to design a detector to recover the transmitted symbol from observation of the $s_{B I}[n]$ samples. The number of samples per symbol is equal to $\operatorname{int}\left(\frac{T}{T_{s}}\right)$. In our particular case, as $\frac{T}{T_{s}}$ is already an integer, the symbol int will be omitted to simplify notation.

Our detection problem consists on, after observing $\frac{T}{T_{s}}$ samples of $s_{B I}[n]$, choosing between two hypothesis: $H_{0}$, if the transmitted symbol was $a_{i}=-1$, and $H_{1}$, if the trasmitted symbol was $a_{i}=1$. We start defining the vector $\mathbf{s}$ :

$$
\mathbf{s}=\left[\begin{array}{llll}
s_{B I}[n] & s_{B I}[n-1] & \cdots & s_{B I}\left[n-\frac{T}{T_{s}}+1\right]
\end{array}\right]^{T}
$$

which is the vector of the samples corresponding to a given symbol. Considering a gaussian and colored noise, the joint probability density functions, with respect to each hypothesis, are:

$$
\begin{aligned}
& p_{\mathbf{s}}\left(\mathbf{s} \mid H_{0}\right)=\frac{\exp \left\{-\frac{1}{2}\left(\mathbf{s}-\mathbf{m}_{\mathbf{0}}\right)^{T} \mathbf{R}^{-1}\left(\mathbf{s}-\mathbf{m}_{\mathbf{0}}\right)\right\}}{(2 \pi)^{T / 2 T_{s}}|\mathbf{R}|^{1 / 2}} \\
& p_{\mathbf{s}}\left(\mathbf{s} \mid H_{1}\right)=\frac{\exp \left\{-\frac{1}{2}\left(\mathbf{s}-\mathbf{m}_{\mathbf{1}}\right)^{T} \mathbf{R}^{-1}\left(\mathbf{s}-\mathbf{m}_{\mathbf{1}}\right)\right\}}{(2 \pi)^{T / 2 T_{s}}|\mathbf{R}|^{1 / 2}}
\end{aligned}
$$

where:

$$
\begin{gathered}
\mathbf{m}_{\mathbf{0}}=\left[\begin{array}{lllll}
-1 & -1 & \cdots & -1
\end{array}\right]^{T} \\
\mathbf{m}_{\mathbf{1}}=\left[\begin{array}{llllll}
1 & 1 & \cdots & 1
\end{array}\right]^{T} \\
\mathbf{R}=\left[\begin{array}{cccccc}
\frac{N_{o}}{2 T_{s}} & \frac{N_{o}}{4 T_{s}} & 0 & 0 & \cdots & 0 \\
\frac{N_{o}}{4 T_{s}} & \frac{N_{o}}{2 T_{s}} & \frac{N_{o}}{4 T_{s}} & 0 & \cdots & 0 \\
0 & \frac{N_{o}}{4 T_{s}} & \frac{N_{o}}{2 T_{s}} & \frac{N_{o}}{4 T_{s}} & \cdots & 0 \\
0 & 0 & \frac{N_{o}}{4 T_{s}} & \frac{N_{o}}{2 T_{s}} & \cdots & 0 \\
\vdots & \vdots & \vdots & \vdots & \ddots & \vdots \\
0 & 0 & 0 & 0 & \cdots & \frac{N_{o}}{2 T_{s}}
\end{array}\right]
\end{gathered}
$$

and $|\mathbf{R}|$ is the covariance matrix determinant. The size of the mean vectors $\mathbf{m}_{\mathbf{0}}$ and $\mathbf{m}_{\mathbf{1}}$ are $\frac{T}{T_{s}} \times 1$, identical to $\mathbf{s}$, and $\mathbf{R}$ is a $\frac{T}{T_{s}} \mathbf{x} \frac{T}{T_{s}}$ matrix.

Detection of transmitted symbols consists in choosing $H_{1}$, if $p_{\mathbf{s}}\left(\mathbf{s} \mid H_{1}\right)>p_{\mathbf{s}}\left(\mathbf{s} \mid H_{0}\right)$, or $H_{0}$, if $p_{\mathbf{s}}\left(\mathbf{s} \mid H_{1}\right)<p_{\mathbf{s}}\left(\mathbf{s} \mid H_{0}\right)$. This can be written as:

$$
p_{\mathbf{s}}\left(\mathbf{s} \mid H_{1}\right) \stackrel{H_{H_{0}}}{\gtrless} p_{\mathbf{s}}\left(\mathbf{s} \mid H_{0}\right)
$$

In pratice, it is easier to work with the log-distribution function rather than the function itself. Hence, applying natural logarithm at both sides of the Equation (30) and rearranging the terms:

$$
\left(\mathbf{s}-\mathbf{m}_{\mathbf{1}}\right)^{T} \mathbf{R}^{-1}\left(\mathbf{s}-\mathbf{m}_{\mathbf{1}}\right)-\left(\mathbf{s}-\mathbf{m}_{\mathbf{0}}\right)^{T} \mathbf{R}^{-1}\left(\mathbf{s}-\mathbf{m}_{\mathbf{0}}\right) \underset{H_{0}}{\stackrel{H_{1}}{\gtrless}} 0
$$

Developing Equation (31), after some algebra, we obtain Equation (32), which is confirmed by making use of the MATLAB symbolic expressions.

$$
\sum_{k=0}^{\frac{T}{T_{s}}-1} s_{B I}[n-k] \underset{H_{0}}{\stackrel{H_{1}}{\gtrless}} 0
$$

That is an interesting result. It suggests that, even though the noise is colored, the best detection strategy is the mean over all samples of each symbol, and once the baseband pulse is rectangular, the optimum detector is clearly a matched filter. Thus the optimum filtering block in Fig. 1 is implemented by a rectangular filter. This result leads to a great simplification of further system performance analysis.

\section{PERformance AnAlysis}

Once the detector is chosen, it is possible to calculate the signal-to-noise ratio at the filter output. The in-phase component of the matched filter output $s_{C}[n]$ is:

$$
s_{C I}[n]=\sum_{k=-\infty}^{\infty} s_{B I}[n] h[n-k]
$$

where $h[n]$ is its impulse response and $s_{B I}[n]$ is the signal at the input of the optimum filter, given by (14). Replacing (14) into (33), $s_{C I}[n]$ is expressed by:

$$
s_{C I}[n]=\sum_{k=-\infty}^{\infty} \hat{A}[k] h[n-k]+\sum_{k=-\infty}^{\infty} v[k] h[n-k]
$$

The symbol detection is performed over the samples of each pulse. For the sake of simplicity, taking this summation over the first received pulse and sampling at the final time instant $n=N_{s}=\frac{T}{T_{s}}-1$, the expression above reduces to:

$s_{C I}\left[N_{s}\right]=\sum_{k=0}^{N_{s}} \hat{A}[k] h\left[N_{s}-k\right]+\sum_{k=0}^{N_{s}} v[k] h\left[N_{s}-k\right]=M+N$

where $M$ relates to the first summation, over the deterministic signal $\hat{A}[n]$, and $N$ to the second one, over the noise. Next step, the calculation of the noise power, which is equal to the variance of $N$, should be performed:

$$
\sigma_{N}^{2}=E\left\{N^{2}\right\}=\sum_{k=0}^{N_{s}} \sum_{i=0}^{N_{s}} \bar{R}_{v}[k-i] h\left[N_{s}-k\right] h\left[N_{s}-i\right]
$$

Replacing (23) into (36) and after a little algebraic manipulation, the noise power becomes:

$$
\sigma_{N}^{2}=\frac{N_{o}}{2 T_{s}}\left(\frac{T}{T_{s}}\right)+\frac{N_{o}}{2 T_{s}}\left(\frac{T}{T_{s}}-1\right)
$$

At our particular case, there is a large number of samples per pulse $\left(T>>T_{s}\right)$, thus the variance reduces to:

$$
\sigma_{N}^{2}=\frac{N_{o}}{2 T_{s}}\left(\frac{2 T}{T_{s}}-1\right) \approx \frac{N_{o} T}{T_{s}^{2}}
$$

The BPSK demodulator bit error rate (BER) is given by:

$$
B E R=Q\left(\sqrt{\frac{M^{2}}{\sigma_{N}^{2}}}\right)
$$

where $\frac{M^{2}}{\sigma_{N}^{2}}$ is the signal-to-noise ratio of the system. Considering the first transmitted symbol was $a_{o}=1$ and recalling that the pulse amplitude is equal to $\sqrt{\frac{T_{s}}{T}}$, the value of $M^{2}$ is:

$$
M^{2}=\left\{\sum_{k=0}^{N_{s}} \hat{A}[k] h\left[N_{s}-k\right]\right\}^{2}=\left\{\sqrt{\frac{T_{s}}{T}} \frac{T}{T_{s}}\right\}^{2}=\frac{T}{T_{s}}
$$


Replacing (36) and (38) into (37):

$$
B E R=Q\left(\sqrt{\frac{T_{s}}{N_{o}}}\right)
$$

The bit energy $E_{b}$ of the demodulated pulse is $\frac{T_{s}}{2}$, hence:

$$
B E R=Q\left(\sqrt{\frac{2 E_{b}}{N_{o}}}\right)
$$

which is the classical result for BPSK demodulation operating in an AWGN channel. The conclusion drawn from this analysis is that even though the noise is colored by the interpolator, the system performance remains as good as the white noise case.

\section{Simulation Results}

In this section, we present some simulation results concerning the proposed demodulator. It was evaluated the bit error rate as a function of the bit energy per noise power density $\left(E_{b} / N_{o}\right)$ ratio, and also it was determined the power spectral density of the colored noise at the interpolator output. The main purpose of these simulations is to confirm the theoretical results presented in the previous sections. As we mentioned before, the simulation was performed in the following conditions: sampling rate $f_{s}=40 \mathrm{MHz}$, IF carrier frequency $f_{F I}=70 M H z$ and bit rate $\frac{1}{T}=1 M H z$.

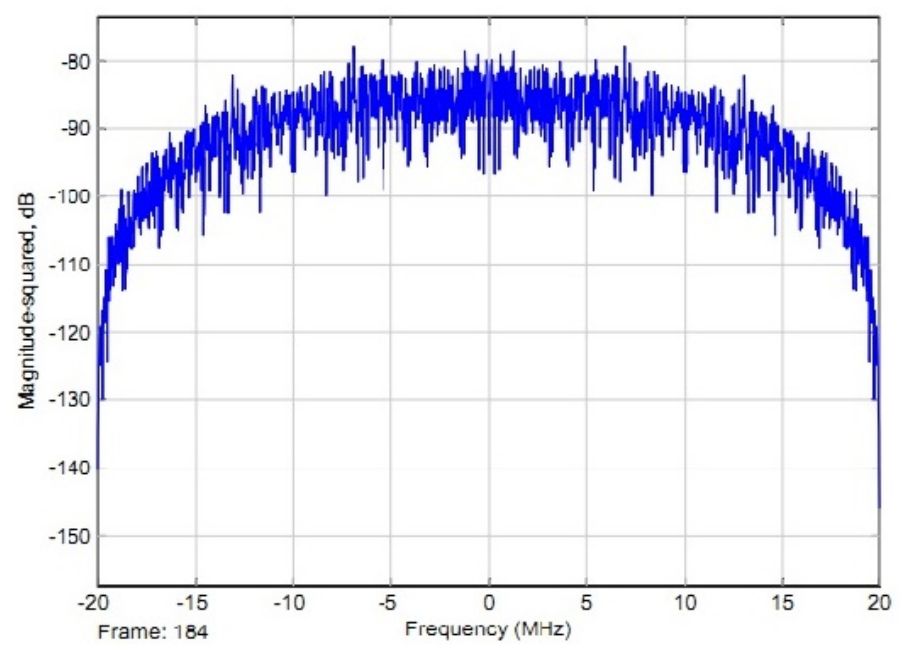

Fig. 3. Power spectral density of the noise $v[n]$.

The power spectral density of $v[n]$ is shown in Fig. 3. Clearly, as it can be seen from its round shape, $v[n]$ is a colored noise, like was predicted in Equation (23). The simulation result of the BER versus $E_{b} / N_{o}$ is presented in Fig. 4, where the implemented model was perfectly synchronized in terms of carrier and symbol timing. Note the system performance is in accordance with Equation (42).

\section{CONCLUSION}

The proposed all-digital BPSK demodulator performs simultaneously sampling and IF to baseband frequency down conversion, utilizing a new interpolator and a new data detector, presenting a very good performance. All the functionalities

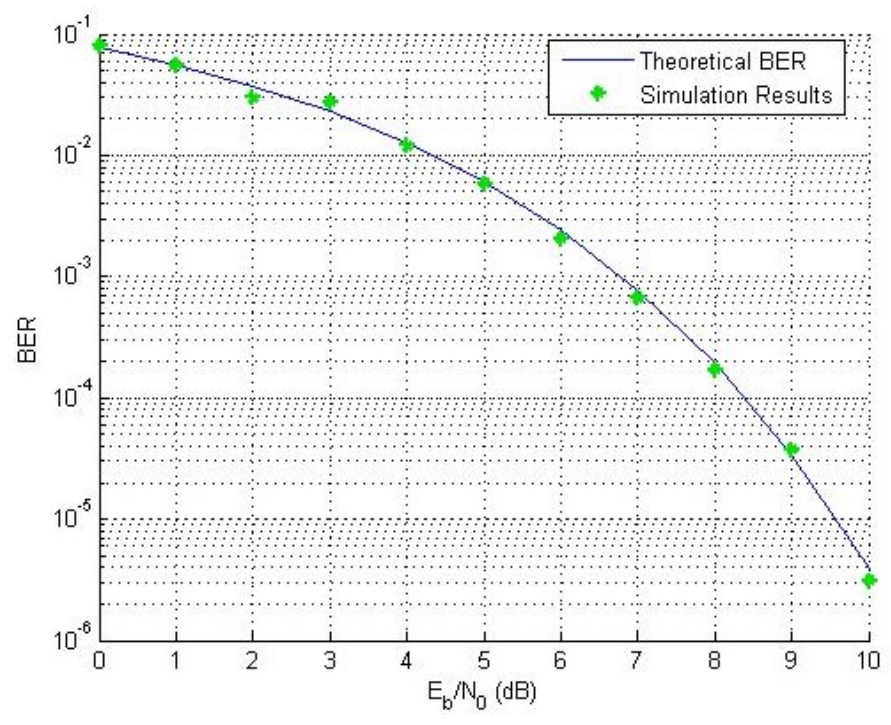

Fig. 4. Bit error rate versus $E_{b} / N_{o}$.

was designed using digital signal processing techniques, discarding the need of analog components at the system. The great contributions of this work are the new interpolation scheme and the design of a ML data detector for non-white noise, as well as all the mathematical analysis. As we have seen by analysis and simulation, the system performace, with this interpolation technique and data detector, is identical to the classical optimum BPSK detection for the AWGN channel.

\section{ACKNOWLEDGMENT}

The authors would like to thank the financial support of CAPES and CNPq. This work was developped under the partnership between the National Institute of Space Research (INPE) and the Federal University of Ceara (UFC).

\section{REFERENCES}

[1] CCSDS Recommendations for Space Data System Standards, Radio Frequency and Modulation Systems - PART 1 Earth Stations and Spacecraft, CCSDS 401.0-b-21tc1. BLUE BOOK, July 2011.

[2] MISHALI, M.; ELDAR, Y. C. Sub-Nyquist Samplig, Bridging theory and practice, IEEE Signal Processing Magazine, November 2011.

[3] SOMBRA,A.; MOTA, J. C. M.; LUCENA, A. M. P. Amostragem em Banda-passante e Conversao de Frequencia em um Demodulador OQPSK Completamente Digital, Anais do XXIX Simposio Brasileiro de Telecomunicacoes SBrT11, Outubro 2011, Curitiba, PR.

[4] OERDER, M. and MEYR H. Digital Filter and Square Timing Recovery, IEEE Transactions on Communications, Vol. COM-36, No. 5, May 1988, pp. 605-612.

[5] ROSHNA, T. R. ; NIVIN, R. ; JOY, S. ; APREN, T.J. ; ALEX, V. Design and Implementation of digital Costas loop and Bit synchronizer in FPGA for BPSK demodulation, Control Communication and Computing (ICCC), 2013 International Conference on.

[6] MEYR, H. et al. Digital Communication Receivers: Synchronization, Channel Estimation, and Signal Processing. JohnWiley and Sons, New York, NY, USA, 1997.

[7] PROAKIS, J. G.; SALEHI, M. Digital Communications, Fifth Edition, McGraw-Hill International Edition. 